\title{
Utility of the Bidirectional Glenn Shunt
}

\section{Mark Nelson Awori, Mohammed Nabil Khan Mohamed, Ali Ahmed Mohamed}

School of Medicine, University of Nairobi

Correspondence to: Dr. Mark Awori, PO Box 14677-00800 Nairobi, Kenya; email: mnawori@yahoo.com

\begin{abstract}
Background: Congenital heart disease (CHD) is a significant cause of childhood morbidity and mortality worldwide. Bidirectional Glenn Shunts (BDGS) form part of the surgical strategy used to treat $\mathrm{CHD}$; no data exists on BDGS usage in the study locality. Methods: A 7-year retrospective, descriptive study was carried out at the Kenyatta National Hospital in Nairobi, Kenya, between 1 January 2006 and 31 December 2012. Results: Eleven BDGS were performed on 11 patients; $63.6 \%$ had tricuspid atresia, $27.3 \%$ had double outlet right ventricle and $9.1 \%$ had pulmonary atresia with intact ventricular septum. Conclusion: Further studies
\end{abstract}

are warranted to identify factors contributing to the late performance of BDGS, poor post-operative follow-up and failure to perform FC.

Key words: Cavopulmonary, Glenn, Shunt, Bidirectional Ann Afr Surg. 2019; 16 (1):30-32

DOI: http://dx.doi.org/10.4314/aas.v16il.7

Conflicts of Interest: None

Funding: None

(C) 2019 Author. This work is licensed under the Creative Commons Attribution 4.0 International License.

\section{Introduction}

Congenital heart disease is the most common congenital abnormality; its prevalence is estimated to be 1:1,000 live births (1). As a result of contemporary interventions, most patients can survive into adulthood (2): the median age at death was 2 years in 1993 and 23 years in the current era (3). From a surgical perspective, CHD may be divided into 3 categories:

1. Patients in whom the lesion can be anatomically corrected surgically and who will not require further surgery

2. Patients in whom the lesion can be anatomically corrected surgically but will require further surgical intervention(s)

3. Patients in whom the lesion can only be palliated surgically.

The palliative surgical solutions offered in earlier eras were not as efficient and durable as the current solutions. Presently, to survive into adulthood, most of the patients in the 3rd category will require a single ventricle to do the work of two. The most significant surgical innovations in this regard were the introduction of the "Classical" Glenn shunt in 1958 (4) and the "Classical" Fontan connection (CFC) in 1971(5). Prior to these two innovations, a systemic arterialto-pulmonary artery shunt such as a Blalock-Taussig shunt (6) was created. It was symptomatically effective but caused significant volume loading of the systemic ventricle with eventual heart failure. The classical Glenn shunt (CGS) involves connecting the superior vena cava (SVC) to the right pulmonary artery alone (4). A subsequent modification (the bidirectional Glenn shunt or BDGS; Figure 1) connects the SVC to both the right and left pulmonary arteries (7). Over time the quality of palliation provided by the CGS and BDGS deteriorates as the relative volume of venous return contributed by the SVC decreases with age. The CFC improves arterial oxygenation by re-routing the inferior vena cava (IVC) to the left pulmonary artery in a patient with a prior CGS.

In contemporary surgery, it is important to allow the child to grow sufficiently before performing the Fontan connection (FC) as this enables an optimal sized prosthetic conduit to be inserted (between the IVC and the right pulmonary artery) (Figure 2) for adequate blood flow as an adult (8). This staging involves performing a BDGS first and completing the FC several years later. Contemporary practice favors performing a BDGS at 3-4 months of age and creating the $\mathrm{FC}$ at 3-4 years of age. This study aimed to determine the indications for and surgical outcomes of BDGS in the study locality.

\section{Methods}

This 7-year retrospective, descriptive study was carried out at the Kenyatta National Hospital (KNH) in Nairobi, Kenya, between 1 January 2006 and 31 December 2012. This was a file-based study; patient case notes were examined and the relevant data was entered into a data sheet.

All patients who underwent a BDGS procedure at $\mathrm{KNH}$ during the study period were included. Variables collected were: type of cardiac lesion, age at surgery, use of 


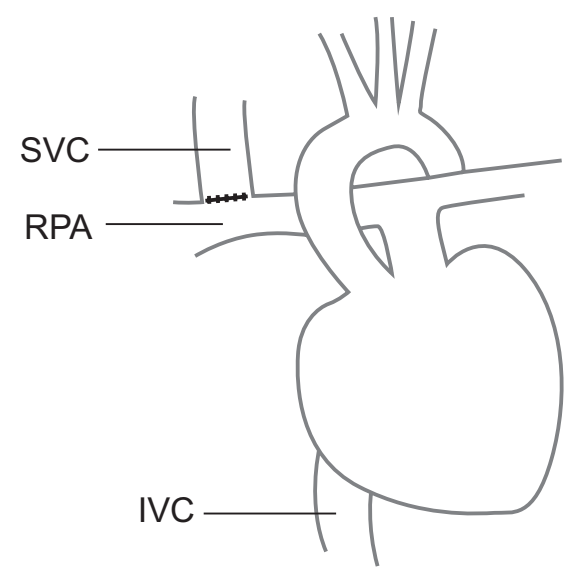

Figure 1: Bidirectional Glenn shunt. (SVC = superior vena cava, RPA = right pulmonary artery, IVC = inferior vena cava)

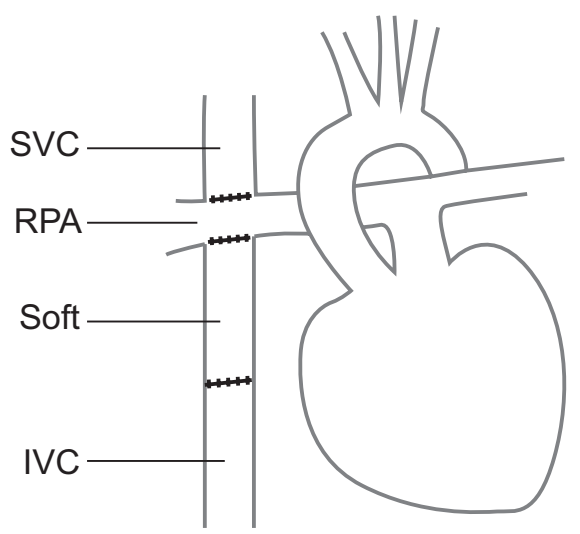

Figure 2: The Fontan connection. (SVC = superior vena cava, RPA = right pulmonary artery, IVC = inferior vena cava)

postoperative anticoagulation, length of intensive care unit stay (LOICUS), operative mortality, length of hospital stay (LOHS), duration of post-operative follow-up and pre- and postoperative arterial oxygen saturations. Microsoft Excel (2010) was used for data analysis. The study was carried out after approval by the Kenyatta National Hospital-University of Nairobi Ethical and Research Committee.

\section{Results}

Eleven BDGS were performed on 11 patients. All surgeries were performed by the resident team. Table 1 summarizes the patient demographics. The operative mortality rate was $9.1 \%(1 / 11)$ and the postoperative follow-up rate was $30 \%$ $(3 / 10)$. Ten out of $11(90.9 \%)$ BDGS were performed under cardiopulmonary bypass. One patient presented with a patent ductus arteriosus (PDA) which was not ligated at surgery. Postoperative heparin was used in 1 patient (9.1\%), and no patients went home on aspirin. The single postoperative mortality was in a 1.5-month old male with tricuspid atresia. No FC procedure was performed. There were three types of lesions: $63.6 \%$ were tricuspid atresia (TA), $27.3 \%$ were double outlet right ventricle (DORV) and 9.1\% were pulmonary atresia with intact ventricular septum (PA-IVS).

Table 1: Patient demographics

\begin{tabular}{ll}
\hline Parameter & Median \\
\hline Age & 36 months \\
Weight & $11.95 \mathrm{~kg}$ \\
Pre-op arterial oxygen saturation & $80 \%$ \\
Post-op arterial oxygen saturation & $91 \%$ \\
Length of intensive care unit stay & 2 days \\
Length of hospital stay & 7 days \\
\hline
\end{tabular}

\section{Discussion}

When CHD cannot be anatomically corrected, most patients can be offered a robust palliative surgical alternative (one of several variants of the CFC). The final result of these variants is that the SVC and IVC venous flow is redirected to the lungs, completely bypassing the right side of the heart. This is achievable because the pulmonary arterial system is a low-resistance and low-pressure system. Neonates, however, have a high resistance and a high-pressure pulmonary arterial system; this falls to adult levels by the 3rd month of life (9). For this reason, a systemic arterial-to-pulmonary artery shunt is performed in patients younger than 3 months of age as the CGS, BSGS or FC will not be tolerated (10).

When the palliative pathway is chosen, the goal is to create a FC. Typically, the ideal FC is achieved by the age of 3 years through 2 or 3 operations. In our study, the median age at BDGS creation was 36 months. This is significant for two reasons: first, this is far older than the recommended 3 months of age; second, a BDGS was created when ideally a FC was indicated. Previous work done in the study locality (11) demonstrated that the mean age at referral to a paediatric cardiologist was 16.9 months and the mean age at confirmation of CHD by echocardiography was 18.6 months. The study also showed that the mean duration from confirmatory echocardiogram to the 1st operation for CHD was 8 months. This work highlights significant delays in both diagnosis and surgery; this may have contributed to the late BDGS in our study. A BDGS was performed instead of a FC even when patients presented late as evidence suggests that the outcome for FC is better when performed in a staged manner (12).

It is striking that no FC were performed. There is longterm (20-30 years) outcome data on CGS (with or without concomitant systemic arterial-to-pulmonary artery shunts) that show results comparable to some FC long-term outcome data in terms of survival (13). It is conceivable that these 
data may have influenced surgical decision making in our resource-constrained environment favoring BDGS over FC. Our $30 \%$ follow-up rate is low. There is anecdotal evidence that patients will not seek medical attention in our region as long as they are feeling reasonably well. BDGS can provide a reasonable quality of life for several years. This too may have contributed to failure to perform FC. Recent long-term FC outcome data are available that show that the contemporary FC offers the best long-term palliation and the dreaded protein-loosing enteropathy occurs in only about $9 \%$ of patients (14).

Our surgical results and the spectrum of lesions palliated are similar to what has been reported elsewhere (10). Only one public hospital regularly performs cardiac surgery in Kenya and it accounts for about half of all cardiac surgeries performed in the country. Six cardiac surgeons work at this institution. It is estimated that over 12,000 children are born in Kenya each year who require cardiac surgery on cardiopulmonary bypass; less than $1 \%$ receive surgery (11). Anecdotal evidence suggests that this is primarily because of inadequate mobilization of resources to deliver this service; the quality of our BDGS service may be a reflection of this.

\section{Conclusion}

Current evidence suggests that FC should be offered where indicated to patients; however, established protocols for optimal palliative surgery for CHD have not been employed in our patients. Further studies are warranted to identify factors contributing to the late performance of the BDGS and failure to perform the $\mathrm{FC}$ in patients.

\section{References}

1. Van der Linde D, Konings EE, Slager MA, et al. Birth prevalence of congenital heart disease worldwide: a systematic review and meta-analysis. J Am Coll Cardio. 2011 Nov 15; 58 (21):2241-7.

2. Krasuski RA, Bashore TM. Congenital heart disease epidemiology in the United States. Blindly feeling for the charging elephant. Circulation. 2016; 134:110-113

3. Khairy P, Ionescu-Ittu R, Mackie AS, et al. Changing mortality in congenital heart disease. J Am Coll Cardiol. 2010; 56:114957.

4. Glenn, WW. Circulatory bypass of the right side of the heartShunt between superior vena cava and distal right pulmonary artery-report of clinical application. NEJM. 1958; 259(3):11720.

5. Fontan F, Baudet E. Surgical repair of tricuspid atresia. Thorax. 1971; 26:240-248.

6. Blalock A, Taussig HB: The surgical treatment of malformations of the heart in which there is pulmonary stenosis or pulmonary atresia. JAMA. 1945; 128:189-202.

7. Hopkins RA, Armstrong BE, Serwer GA, et al. Physiologic rationale for a bidirectional cavopulmonary shunt; a versatile component to the Fontan principle. J Thorac Cardiovasc Surg. 1985; 90:391-398.

8. Marcelletti C, Corno A, Giannico S, et al. Inferior vena cavapulmonary artery extracardiac conduit. A new form of right heart bypass. J Thorac Cardiovasc Surg. 1990; 100:228-232.

9. Rudolph AM. The changes in the circulation after birth-their importance in congenital heart disease. Circulation 1970; 41:343-349.

10. Bradley SM, Mosca RS, Hennein HA, et al. Bidirectional superior cavopulmonary connection in young infants. Circulation 1996; 94(Suppl II):5-11.

11. Awori MN, Ogendo SW, Gitome SW, et al. Management pathway for congenital heart disease at Kenyatta National Hospital, Nairobi. East Afr Med J. 2007; 84(7):312-317.

12. Tamagond S, Agarwal S, Prakash S, et al. Single stage versus two stage repair for univentricular heart-our experience. Indian J Thorac Cardiovasc Surg. 2011; 27(2):83-87.

13. Yeh Jr T, Williams WG, McCrindle BW, et al. Equivalent survival following cavopulmonary shunt: with or without the Fontan procedure. Eur J Cardiothorac Surg. 1999 Aug; 16(2):111-6.

14. Pundi KN, Johnson JN, Dearani JA, et al. 40-year follow-up after the Fontan operation. Long-term outcomes of 1,052 Patients. J Am Coll Cardiol. 2015; 66(15):1700-10. 